\title{
The importance of communicating histories of gender assignment and reassignment to genetic laboratories
}

\author{
Jess F. Peterson, MD¹, Peggy J. Andringa, BA ${ }^{2}$, John W. Grignon Jr, BS² and Peter vanTuinen, PhD $^{1}$
}

Advancements in medicine have allowed for the medical or surgical modification of ambiguous genitalia, or for sex reassignment in transpeople. ${ }^{1-4}$ The etiology of ambiguous genitalia is broad, including genetic alterations, hormone imbalances, gonadal dysgenesis, or structural abnormalities. ${ }^{2,5}$ Depending on the presence of XY or XX chromosomes, gender assignment is largely dependent on the etiology of ambiguous genitalia. ${ }^{2}$ For example, the recommended sex assignment for $46, \mathrm{XX}$ with congenital adrenal hyperplasia or $46, \mathrm{XY}$ and complete gonadal dysgenesis is female, while the recommended sex assignment for 46,XY with 5-alphareductase- 2 deficiency could be male or female depending on sexual potential. ${ }^{2}$ Gender identity indicates the persistent sense of self as a woman or man and, as such, transpeople who identify as the opposite sex may pursue gender reassignment by means of hormone replacement therapy, surgery, or a combination of both. ${ }^{1,2}$ However, the complexity of gender identity is increasingly perceived as a bimodal continuum instead of a binary categorical system. ${ }^{4}$ Several countries, including India, Nepal, Pakistan, and Bangladesh, recognize a third nonmale/nonfemale gender category, and this is listed as such on official government documents. ${ }^{4}$

Importantly, gender reassignment will result in a discrepancy between genetic sex $(46, \mathrm{XX}$ or $46, \mathrm{XY})$ and gender identity (female or male), while gender assignment could result in a discrepancy between genetic sex and gender identity. Our laboratory has recently encountered genetic sex and gender identity discrepancies on two occasions, both of which led to extensive laboratory investigations only to discover that sex assignment or reassignment had occurred. Importantly, this information was not indicated on the test requisition forms. Both patients had histories of lymphoma and underwent bone marrow biopsies for staging purposes. Conventional cytogenetics were performed and the genetic sex was opposite to the sex indicated on the test requisition forms. These discrepancies prompted additional investigations to rule out several possibilities including: specimen mixup from the referring institution; specimen mislabeling in our laboratory; potential specimen mix-ups at various stages of metaphase cell preparation; and histories of opposite sex bone marrow transplants. After exhausting all these explanations for the discrepancy in our first case, our discussion with the referring clinician revealed a history of ambiguous genitalia and gender assignment surgery. For the second case, a thorough search through the patient history revealed femaleto-male gender reassignment.

Errors in laboratory medicine are traditionally categorized by pre-analytical, analytical, and post-analytical phases. ${ }^{6-8}$ Further subdividing the preanalytical phase, "conventional" preanalytical errors occur under the control of the laboratory, while pre-pre-analytical errors occur outside of the laboratory and can result from test ordering practices, specimen collecting and handling, or transportation. ${ }^{6}$ Genetic sex and gender identity discrepancies encountered in genetic laboratories can certainly be classified as prepreanalytical errors and can have several unfavorable outcomes. First and most importantly, failure to communicate a history of gender assignment or reassignment could pose a serious risk to patient care. For example, risk calculations or recommendations for a patient with a $B R C A 1 / 2$ mutation could be inaccurate if the family history fails to identify a transgender family member by their genetic sex rather than by gender identity. ${ }^{9}$ Some reference ranges for analytes (e.g., hemoglobin, hematocrit, lipid profiles) in clinical chemistry and hematology are also based on genetic sex, and discrepancies could cause unnecessary evaluation, potential misdiagnosis, and unneeded therapy. ${ }^{10}$ In addition, troubleshooting major discrepancies in genetic laboratories require a great deal of time and resources. Effectively, communicating a history of gender assignment or reassignment would prevent laboratory personal from spending an exorbitant amount of time investigating discrepant results.

One of several critical aspects identified in the pre-preanalytical phase includes "the right patient", and addresses issues associated with patient identification. ${ }^{8}$ Avoiding specimen mix-up by using multiple identifiers (e.g., date of birth, name, sex, medical record number) has greatly reduced the risk of errors and is applicable to the majority of specimens handled in laboratory medicine. However, if gender assignment or reassignment has occurred, a potential patient

${ }^{1}$ Department of Pathology, Medical College of Wisconsin, Milwaukee, Wisconsin, USA; ${ }^{2}$ Wisconsin Diagnostic Laboratories, Milwaukee, Wisconsin, USA. Correspondence: Jess F. Peterson (jepeterson@mcw.edu)

Submitted 30 December 2016; accepted 12 March 2017; advance online publication 22 June 2017. doi:10.1038/gim.2017.44 


\section{COMMENTARY}

identifier discrepancy may occur (sex reported on the test requisition form versus genetic sex). We have identified several potential solutions to address this issue. Substituting "birth sex" for "sex" on test requisition forms would address those that underwent gender assignment (if the genetic sex is known) or reassignment. Check boxes indicating a history of ambiguous genitalia or gender reassignment could also be incorporated into the test requisition form. Perhaps the most reasonable solution is fostering open and frequent communication between clinicians and laboratory directors. By doing so, laboratory directors could emphasize the importance of communicating histories of gender assignment and reassignment and how they may impact patient care and laboratory operations.

In conclusion, gender assignment and reassignment can lead to patient identifier discrepancies that, if not effectively communicated to the genetics laboratory, could potentially compromise patient care or lead to extensive and unnecessary laboratory troubleshooting. Several options have been proposed that may facilitate gathering this information more readily-most importantly, open and frequent communication between clinicians and laboratory directors.
PETERSON et al | Communicating histories of gender assignment or reassignment

\section{DISCLOSURE}

Jess F. Peterson currently serves on the American College of Medical Genetics and Genomics Membership Committee.

\section{REFERENCES}

1. Castellano E, Crespi C, Dell'Aquila C, et al. Quality of life and hormones after sex reassignment surgery. J Endocrinol Invest 2015;38:1373-1381.

2. Fisher $A D$, Ristori J, Fanni $E$, et al. Gender identity, gender assignment and reassignment in individuals with disorders of sex development: a major of dilemma. J Endocrinol Invest 2016;39:1207-1224.

3. Lee PA, Nordenström A, Houk CP, et al. Global disorders of sex development update since 2006: perceptions, approach and care. Horm Res Paediatr 2016:85:158-180

4. Meyer-Bahlburg HF, Baratz Dalke K, Berenbaum SA, et al. Gender assignment, reassignment and outcome in disorders of sex development: update of the 2005 Consensus Conference. Horm Res Paediatr 2016;85:112-118.

5. Bashamboo A, McElreavey K. Mechanism of sex determination in humans: insights from disorders of sex development. Sex Dev 2016;10:313-325.

6. Da Rin G. Pre-analytical workstations: a tool for reducing laboratory errors. Clin Chim Acta 2009;404:68-74.

7. Gersen SL, Keagle MB (eds). The Principles of Clinical Cytogenetics, 3rd edn. Springer: New York, 2013.

8. Plebani M. Towards a new paradigm in laboratory medicine: the five rights. Clin Chem Lab Med 2016;54:1881-1891.

9. Katapodi MC, DeFlon SL, Milliron KJ, et al. Knowledge of risk factors and modes of gene transmission among women at risk for hereditary breast and ovarian cancer syndrome. J Clin Oncol 2011;29:e12017.

10. Roberts TK, Kraft CS, French D, et al. Interpreting laboratory results in transgender patients on hormone therapy. Am J Med 2014;127: 159-162. 